\title{
LETTER
}

\section{Sr and Nd isotope ratios of twelve GSJ rock reference samples}

\author{
TAKASHI MIYAZAKI ${ }^{1}$ and KenJi ShUto ${ }^{2}$ \\ ${ }^{1}$ Graduate School of Science and Technology, Niigata University, Ikarashi 2-8050, Niigata 950-2181, Japan \\ ${ }^{2}$ Department of Geology, Faculty of Science, Niigata University, Ikarashi 2-8050, Niigata 950-2181, Japan
}

(Received September 5, 1997; Accepted June 24, 1998)

\begin{abstract}
${ }^{87} \mathrm{Sr} /{ }^{86} \mathrm{Sr}$ and ${ }^{143} \mathrm{Nd} /{ }^{144} \mathrm{Nd}$ ratios for twelve GSJ rock reference samples (GSJ-RRS), together with the chemical standards NBS987, La Jolla and JNdi-1, have been measured on a MAT262 mass spectrometer in the Department of Geology, Niigata University. Measurements of five to nine aliquots for each GSJRRS gave small standard deviations of both $\mathrm{Sr}$ and $\mathrm{Nd}$ isotopic ratios.
\end{abstract}

\section{INTRODUCTION}

The standard samples of "igneous rock series" provided by the Geological Survey of Japan (GSJ rock reference samples, GSJ-RRS) are widely used for analytical references of geological samples, and their $\mathrm{Sr}$ and $\mathrm{Nd}$ isotopic ratios have been reported from several laboratories (e.g., Shibata and Adachi, 1972; Nohda and Wasserburg, 1981; Kagami et al., 1982, 1987, 1989; Kurasawa, 1984; Zhang Zichao, 1987; Ando and Shibata, 1988; Okano et al., 1989; Arakawa, 1992; Iizumi et al., 1994, 1995; Yamamoto and Maruyama, 1996). However, there are still few isotopic data for some GSJ-RRS, and $\mathrm{Nd}$ isotope ratios have not yet been reported for some samples, such as JA-3 and JR-2. Also, as pointed out by some authors (e.g., Kagami et al., 1989; Arakawa, 1992), some GSJ-RRS exhibit systematic differences in $\mathrm{Sr}$ and $\mathrm{Nd}$ isotopic ratios between laboratories even if normalization has been done using standard samples of chemicals.

We measured ${ }^{87} \mathrm{Sr} /{ }^{86} \mathrm{Sr}$ and ${ }^{143} \mathrm{Nd} /{ }^{144} \mathrm{Nd}$ ratios for JB-1a, JB-2, JB-3, JA-1, JA-2, JA-3, JG-1a, JG-3, JGb-1 and JR-1, ${ }^{87} \mathrm{Sr} /{ }^{86} \mathrm{Sr}$ ratios for JF-1 and $\mathrm{JF}-2$, and ${ }^{143} \mathrm{Nd} /{ }^{144} \mathrm{Nd}$ ratios for JG-2 and JR-2. Strontium and $\mathrm{Nd}$ isotopic ratios were measured for five to nine separate aliquots of each GSJRRS. These data were compared with previously reported data which were normalized using chemical standards.

\section{Analytical Procedures}

Separation of $\mathrm{Sr}$ and $\mathrm{Nd}$ was carried out in the Department of Geology, Niigata University, essentially following the method described by Kagami et al. (1982, 1987). Each powdered sample (about $100 \mathrm{mg}$ ) was decomposed in a sealed teflon vessel using a $\mathrm{HF}, \mathrm{HCl}, \mathrm{HNO}_{3}$ and $\mathrm{HClO}_{4}$ mixture. Five to nine decomposed samples were prepared for each GSJ-RRS and extraction of $\mathrm{Sr}$ and $\mathrm{Nd}$ from these samples were made using different columns filled with cation exchange resin (Dowex AG 50W-X8, 200-400 mesh). The extracted $\mathrm{Sr}$ and $\mathrm{Nd}$ were loaded on Ta filaments with $2.5 \mathrm{~N} \mathrm{HCl}$ and $2.5 \mathrm{~N}, \mathrm{HNO}_{3}$, respectively.

The measurements were made with a Ta filament for evaporation and Re filament for ionization, using a Finnigan MAT 262 thermal ionization mass spectrometer equipped with nine Faraday collectors, which was installed in the Department of Geology, Niigata University. The data were obtained in so-called "static mode" in which ion beam of each mass number is collected by a fixed Faraday cup. These data were computed from 150 500 repeated measurements, comprising ten scans 
in each of ten to fifty blocks.

The measured ${ }^{87} \mathrm{Sr} /{ }^{86} \mathrm{Sr}$ ratios were normalized to a ${ }^{86} \mathrm{Sr} /{ }^{88} \mathrm{Sr}$ ratio of 0.1194 , and the measured ${ }^{143} \mathrm{Nd} /{ }^{144} \mathrm{Nd}$ to a ${ }^{146} \mathrm{Nd} /{ }^{144} \mathrm{Nd}$ ratio of 0.7219 . Since ${ }^{85} \mathrm{Rb}$ and ${ }^{147} \mathrm{Sm}$ were not detected during the course of this study, contribution from ${ }^{87} \mathrm{Rb}$ and ${ }^{144} \mathrm{Sm}$ were negligible. The total blanks of $\mathrm{Sr}$ and $\mathrm{Nd}$ in the whole procedure are less than $0.4 \mathrm{ng}$ and $0.3 \mathrm{ng}$, respectively.

\section{RESUlts}

${ }^{87} \mathrm{Sr}{ }^{86} \mathrm{Sr}$ and ${ }^{143} \mathrm{Nd} /{ }^{144} \mathrm{Nd}$ ratios of the standard chemical samples, NBS987, La Jolla and JNdi-1

The NBS987 standard determined during this study gave a mean ${ }^{87} \mathrm{Sr} /{ }^{86} \mathrm{Sr}$ ratio of $0.710251 \pm$ $0.000004\left(2 \sigma_{\mathrm{m}}, N=51\right)$. During the course of this study, we also measured ${ }^{143} \mathrm{Nd} /{ }^{144} \mathrm{Nd}$ ratios of two standard samples, La Jolla and JNdi-1. The latter a new standard provided by Geological Survey of Japan. The La Jolla standard gave a mean ${ }^{143} \mathrm{Nd} /$ ${ }^{144} \mathrm{Nd}$ ratio of $0.511851 \pm 0.000006\left(2 \sigma_{\mathrm{m}}, N=17\right)$ whereas JNdi-1 gave a mean ${ }^{143} \mathrm{Nd} /{ }^{144} \mathrm{Nd}$ ratio of $0.512106 \pm 0.000003\left(2 \sigma_{\mathrm{m}}, N=44\right)$. Tanaka et al. (1997) reported ${ }^{143} \mathrm{Nd} /{ }^{144} \mathrm{Nd}$ ratio of $0.512116 \pm$ 0.000008 for standard sample JNdi-1, which was corrected by normalizing to La Jolla as reported in Lugmair and Carlson (1978). Our mean ${ }^{143} \mathrm{Nd} /$ ${ }^{144} \mathrm{Nd}$ ratio for the JNdi- 1 standard corrected in the same manner gives $0.512113 \pm 0.000003$, which is almost identical to the value of Tanaka et al. (1997).

${ }^{87} \mathrm{Sr} /{ }^{86} \mathrm{Sr}$ and ${ }^{143} \mathrm{Nd} /{ }^{144} \mathrm{Nd}$ ratios of GSJ rock reference samples

Strontium isotopic ratios of five or eight separately decomposed samples of each GSJ-RRS are listed with average values and the standard deviations in Table 1. Kurasawa (1984), Zhang Zichao (1987), Kagami et al. (1989), Iizumi et al. (1994) and Yamamoto and Maruyama (1996) also reported $\mathrm{Sr}$ isotope values of GSJ-RRS with the value of NBS987. The NBS987 values in these reports are $0.710278 \pm 0.000009\left(2 \sigma_{\mathrm{m}}\right), 0.71030 \pm$ $0.00003\left(2 \sigma_{\mathrm{m}}\right), 0.710252 \pm 0.000018\left(2 \sigma_{\mathrm{m}}\right)$, $0.710275 \pm 0.000007\left(2 \sigma_{\mathrm{m}}\right)$ and $0.710241 \pm$ $0.000022\left(2 \sigma_{\mathrm{m}}\right)$, respectively. Hereafter, we will compare our data on GSJ-RRS to the previously reported data normalized using NBS987 values of 0.710251 .

Eight separate aliquots of JB-1a gave an average ${ }^{87} \mathrm{Sr} /{ }^{86} \mathrm{Sr}$ ratio of $0.704107 \pm 0.000008\left(2 \sigma_{\mathrm{m}}\right)$. Previously reported values for JB-1a exhibit a large variation from 0.704082 to 0.70415 (Zhang Zichao, 1987; Kagami et al., 1989; Iizumi et al., 1994; Yamamoto and Maruyama, 1996). Our value is slightly higher than that of $0.704082 \pm 0.000016$ $\left(2 \sigma_{\mathrm{m}}\right)$ reported by Kagami et al. (1989), but very close to the value of $0.704109 \pm 0.000007\left(2 \sigma_{\mathrm{m}}\right)$ by Iizumi et al. (1994) and the value of 0.704116 $\pm 0.000012\left(2 \sigma_{\mathrm{m}}\right)$ by Yamamoto and Maruyama (1996).

Mean ${ }^{87} \mathrm{Sr} /{ }^{86} \mathrm{Sr}$ ratios of JB-2, JA-1 and JG-1a are within the range of previously reported data (JB-2; 0.703667 0.703727; Kurasawa, 1984; Zhang Zichao, 1987; Iizumi et al., 1994, JA-1; 0.703506 0.70362; Kurasawa, 1984; Zhang Zichao, 1987; Kagami et al., 1989; Iizumi et al., 1994, JG-1a; 0.71093 0.710972; Zhang Zichao, 1987; Kagami et al., 1989; Iizumi et al., 1994; Yamamoto and Maruyama, 1996). Mean ${ }^{87} \mathrm{Sr} /{ }^{86} \mathrm{Sr}$ ratios of JB-3 and JR-1 are within the deviation of the lowest values previously reported (JB-3; $0.703415 \pm 0.000005\left(2 \sigma_{\mathrm{m}}\right)$; Iizumi et al., 1994, JR-1;0.70413 $\pm 0.00003\left(2 \sigma_{\mathrm{m}}\right)$; Zhang Zichao, 1987). A mean ${ }^{87} \mathrm{Sr} /{ }^{86} \mathrm{Sr}$ ratio of JA-3 is close to the lowest value previously reported $(0.704173 \pm$ $0.000009\left(2 \sigma_{\mathrm{m}}\right)$; Iizumi et al., 1994). Mean ${ }^{87} \mathrm{Sr} /$ ${ }^{86} \mathrm{Sr}$ ratios of JA-2, JF-1 and JGb-1 obtained by us are within the deviation of reported values (JA$2 ; 0.70632 \pm 0.00003\left(2 \sigma_{\mathrm{m}}\right), \mathrm{JF}-1 ; 0.71353 \pm$ $0.00004\left(2 \sigma_{\mathrm{m}}\right)$; Zhang Zichao, 1987, JGb-1; $0.705220 \pm 0.000017\left(2 \sigma_{\mathrm{m}}\right)$; Kurasawa, 1984). Our mean $\mathrm{Sr}$ isotopic data for JG-3 and JF-2 are lower than values reported by Zhang Zichao (1987) (JG$3 ; 0.70547 \pm 0.00005\left(2 \sigma_{\mathrm{m}}\right), \mathrm{JF}-2 ; 0.70982 \pm$ $\left.0.00002\left(2 \sigma_{\mathrm{m}}\right)\right)$.

Neodymium isotopic ratios of analyzed samples are listed in Table 2. Our data in Table 2 show ${ }^{143} \mathrm{Nd} /{ }^{144} \mathrm{Nd}$ ratios of either five or nine separate analyses for each reference sample and their average values with the standard deviations. Arakawa 
Table 1. Analytical results for ${ }^{87} \mathrm{Sr} / 86 \mathrm{Sr}$ ratios of twelve GSJ rock reference samples and standard sample NBS987

\begin{tabular}{|c|c|c|c|c|c|c|c|c|c|}
\hline Samples & $" \mathrm{Sr} /{ }^{\circ} \mathrm{Sr} \pm 2 \sigma \mathrm{m}$ & & & & Samples & $" \mathrm{Sr} /{ }^{\mu} \mathrm{Sr} \pm 2 \sigma \mathrm{m}$ & & & \\
\hline $\mathrm{JB} 1 \mathrm{a} \cdot 1$ & 0.704117 & \pm 4 & & & $J G 1 a \cdot 1$ & 0.710962 & \pm 5 & & \\
\hline $\mathrm{JB} 1 \mathrm{a} \cdot 2$ & 0.704115 & \pm 4 & $(n=2)^{\circ}$ & & $\mathrm{JG} 1 \mathrm{a} \cdot 2$ & 0.710967 & \pm 8 & & \\
\hline $\mathrm{JB} 1 \mathrm{a} \cdot 3$ & 0.704119 & \pm 3 & & & $\mathrm{JG} 1 \mathrm{a} \cdot 3$ & 0.710964 & \pm 6 & & \\
\hline$J B 1 a \cdot 4$ & 0.704101 & \pm 7 & $(n=6)^{\circ}$ & & $\mathrm{JGla} \cdot 4$ & 0.710989 & \pm 7 & & \\
\hline $\mathrm{JB} 1 \mathrm{a} \cdot 5$ & 0.704097 & \pm 5 & $(n=2)^{\circ}$ & & $\mathrm{JGla} \cdot 5$ & 0.710967 & \pm 6 & & \\
\hline $\mathrm{JB} 1 \mathrm{a} \cdot 6$ & 0.704094 & \pm 5 & $(n=2)^{\circ}$ & & & 0.710970 & \pm 11 & (s.d.) & $(N=5)^{* *}$ \\
\hline JB1a:7 & 0.704102 & \pm 5 & $(n=2)^{\circ}$ & & & & \pm 13 & $(2 \sigma \mathrm{m})$ & $(N=5)^{*}$ \\
\hline \multirow[t]{5}{*}{$\mathrm{JBla} \cdot 8$} & 0.704110 & \pm 8 & & & & & & & \\
\hline & 0.704107 & \pm 10 & (s.d.) & $(\mathrm{N}=8)^{* *}$ & & & & & \\
\hline & & \pm 8 & $(2 \sigma \mathrm{m})$ & $(\mathrm{N}=8)^{* *}$ & $J G 3 \cdot 1$ & 0.705350 & \pm 6 & & \\
\hline & & & & & $\mathrm{JG} 3 \cdot 2$ & 0.705379 & \pm 8 & & \\
\hline & & & & & $\mathrm{JG} 3 \cdot 3$ & 0.705352 & \pm 6 & & \\
\hline JB2-1 & 0.703673 & \pm 5 & $(n=2)^{\circ}$ & & $\mathrm{JG} 3.4$ & 0.705373 & \pm 5 & & \\
\hline $\mathrm{JB} 2 \cdot 2$ & 0.703679 & \pm 8 & & & JG3.5 & 0.705344 & \pm 5 & & \\
\hline $\mathrm{JB} 2 \cdot 3$ & 0.703657 & \pm 6 & & & & 0.705360 & \pm 15 & (s.d.) & $(N=5)^{* \prime}$ \\
\hline $\mathrm{JB} 2 \cdot 4$ & 0.703663 & \pm 5 & & & & & \pm 19 & $(2 \sigma \mathrm{m})$ & $(\mathrm{N}=5)^{* *}$ \\
\hline \multirow[t]{5}{*}{$\mathrm{JB} 2.5$} & 0.703665 & \pm 6 & & & & & & & \\
\hline & 0.703668 & \pm 9 & (s.d.) & $(\mathrm{N}=5)^{* *}$ & & & & & \\
\hline & & \pm 11 & $(2 \sigma \mathrm{m})$ & $(N=5)^{* *}$ & JGb1.1 & 0.705225 & \pm 10 & & \\
\hline & & & & & JGb1-2 & 0.705204 & \pm 9 & & \\
\hline & & & & & $\mathrm{JGb1} \cdot 3$ & 0.705205 & \pm 7 & & \\
\hline JB3.1 & 0.703406 & \pm 5 & $(n=2)^{\circ}$ & & $\mathrm{JGbl} \cdot 4$ & 0.705196 & \pm 5 & & \\
\hline JB3-2 & 0.703412 & \pm 5 & $(n=2)^{\circ}$ & & $\mathrm{JGb1} .5$ & 0.705195 & \pm 5 & & \\
\hline JB3.3 & 0.703407 & \pm 5 & $(n=2)^{\circ}$ & & & 0.705205 & \pm 12 & (s.d.) & $(\mathrm{N}=5)^{* *}$ \\
\hline JB3-4 & 0.703410 & \pm 5 & $(n=2)^{\circ}$ & & & & \pm 15 & $(2 \sigma \mathrm{m})$ & $(N=5)^{* *}$ \\
\hline \multirow[t]{5}{*}{ JB3-5 } & 0.703416 & \pm 5 & $(n=2)^{\circ}$ & & & & & & \\
\hline & 0.703410 & \pm 4 & (s.d.) & $(\mathrm{N}=5)^{* *}$ & & & & & \\
\hline & & \pm 5 & $(2 \sigma \mathrm{m})$ & $(N=5)^{* *}$ & $J R 1 \cdot 1$ & 0.704127 & \pm 13 & & \\
\hline & & & & & $\mathrm{JR} 1 \cdot 2$ & 0.704125 & \pm 8 & & \\
\hline & & & & & $\mathrm{JR} 1 \cdot 3$ & 0.704119 & \pm 7 & & \\
\hline$J A 1 \cdot 1$ & 0.703546 & \pm 8 & $(n=2)^{\circ}$ & & $J R 1.4$ & 0.704138 & \pm 8 & & \\
\hline$J A 1-2$ & 0.703537 & \pm 5 & $(n=2)^{\circ}$ & & JR1.5 & 0.704123 & \pm 7 & & \\
\hline $\mathrm{JA1} \cdot 3$ & 0.703548 & \pm 7 & $(n=2)^{\circ}$ & & & 0.704127 & \pm 7 & (s.d.) & $(\mathrm{N}=5)^{* *}$ \\
\hline JA1.4 & 0.703541 & \pm 5 & $(n=2)^{\circ}$ & & & & \pm 9 & $(2 \sigma \mathrm{m})$ & $(\mathrm{N}=5)^{* *}$ \\
\hline \multirow[t]{5}{*}{$\mathrm{JA1} .5$} & 0.703541 & \pm 5 & $(n=2)^{\circ}$ & & & & & & \\
\hline & 0.703543 & \pm 4 & (s.d.) & $(N=5)^{* *}$ & & & & & \\
\hline & & \pm 6 & $(2 \sigma \mathrm{m})$ & $(\mathrm{N}=5)^{* *}$ & JF1-1 & 0.713482 & \pm 8 & & \\
\hline & & & & & JF1-2 & 0.713500 & \pm 9 & & \\
\hline & & & & & $\mathrm{JF} 1 \cdot 3$ & 0.713492 & \pm 8 & & \\
\hline JA2-1 & 0.706329 & \pm 5 & $(n=2)^{\circ}$ & & $\mathrm{JF} 1 \cdot 4$ & 0.713501 & \pm 8 & & \\
\hline JA2.2 & 0.706339 & \pm 5 & $(n=2)^{\circ}$ & & $\mathrm{JF} 1.5$ & 0.713487 & \pm 9 & & \\
\hline JA2-3 & 0.706342 & \pm 5 & $(n=2)^{\circ}$ & & & 0.713493 & \pm 8 & (s.d.) & $(\mathrm{N}=5)^{* *}$ \\
\hline JA2-4 & 0.706326 & \pm 5 & & & & & \pm 10 & $(2 \sigma \mathrm{m})$ & $(\mathrm{N}=5)^{* *}$ \\
\hline \multirow[t]{5}{*}{ JA2.5 } & 0.706335 & \pm 5 & & & & & & & \\
\hline & 0.706334 & \pm 7 & (s.d.) & $(\mathrm{N}=5)^{* *}$ & & & & & \\
\hline & & \pm 8 & $(2 \sigma \mathrm{m})$ & $(\mathrm{N}=5)^{* *}$ & $\mathrm{JF} 2 \cdot 1$ & 0.709709 & \pm 8 & & \\
\hline & & & & & JF2-2 & 0.709699 & \pm 8 & & \\
\hline & & & & & $\mathrm{JF} 2 \cdot 3$ & 0.709703 & \pm 8 & & \\
\hline JA3-1 & 0.704163 & \pm 5 & $(n=2)^{*}$ & & JF2.4 & 0.709702 & \pm 9 & & \\
\hline JA3-2 & 0.704147 & \pm 6 & & & $\mathrm{JF} 2.5$ & 0.709710 & \pm 7 & & \\
\hline JA3.3 & 0.704161 & \pm 5 & $(n=2)^{\circ}$ & & & 0.709705 & \pm 5 & (s.d.) & $(N=5)^{* *}$ \\
\hline JA3-4 & 0.704165 & \pm 8 & $(n=2)^{\circ}$ & & & & \pm 6 & $(2 \sigma \mathrm{m})$ & $(\mathrm{N}=5)^{*}$ \\
\hline \multirow[t]{3}{*}{ JA3.5 } & 0.704165 & \pm 6 & & & & & & & \\
\hline & 0.704160 & \pm 8 & (s.d.) & $(\mathrm{N}=5)^{* *}$ & & & & & \\
\hline & & \pm 10 & $(2 \sigma \mathrm{m})$ & $(\mathrm{N}=5)^{* *}$ & NBS987 & 0.710251 & \pm 4 & $(n=51)^{\circ}$ & \\
\hline
\end{tabular}

*Number of measurements; **Number of samples which are separately decomposed and extracted. s.d.: standard deviation. 
Table 2. Analytical results for ${ }^{143} \mathrm{Nd} /{ }^{144} \mathrm{Nd}$ ratios of twelve GSJ rock reference samples and standard samples La Jolla and JNdi-I

\begin{tabular}{|c|c|c|c|c|c|c|c|}
\hline Samples & ${ }^{103} \mathrm{Nd} /{ }^{\prime 4} \mathrm{Nd} \pm 2$ & & & Samples & ${ }^{10} \mathrm{Nd} /{ }^{104} \mathrm{Nd} \pm 2 \sigma \mathrm{m}$ & & \\
\hline $\mathrm{JB} 1 \mathrm{a} \cdot 1$ & 0.512779 & \pm 9 & & $J G 1 a \cdot 1$ & $0.512385 \pm 12$ & & \\
\hline $\mathrm{JB} 1 \mathrm{a} \cdot 2$ & 0.512795 & \pm 10 & & $\mathrm{JG} 1 \mathrm{a} \cdot 2$ & $0.512378 \pm 12$ & & \\
\hline $\mathrm{JB} 1 \mathrm{a} \cdot 3$ & 0.512789 & \pm 9 & & $\mathrm{JGla} \cdot 3$ & $0.512373 \pm 6$ & & \\
\hline $\mathrm{JB} 1 \mathrm{a} \cdot 4$ & 0.512781 & \pm 9 & & $\mathrm{JG1a} \cdot 4$ & $0.512392 \pm 10$ & & \\
\hline $\mathrm{JB} 1 \mathrm{a} \cdot 5$ & 0.512771 & \pm 11 & & $\mathrm{JG} 1 \mathrm{a} \cdot 5$ & $0.512386 \pm 13$ & & \\
\hline $\mathrm{JB} 1 \mathrm{a} \cdot 6$ & 0.512779 & \pm 10 & & & $0.512383 \pm 7$ & (s.d.) & $(\mathrm{N}=5)^{* *}$ \\
\hline JB1a:7 & 0.512785 & \pm 9 & & & \pm 9 & $(2 \sigma \mathrm{m})$ & $(\mathrm{N}=5)^{* *}$ \\
\hline $\mathrm{JB} 1 \mathrm{a} \cdot 8$ & 0.512785 & \pm 9 & & & & & \\
\hline \multirow[t]{5}{*}{$\mathrm{JB} 1 \mathrm{a} \cdot 9$} & 0.512773 & \pm 11 & & & & & \\
\hline & 0.512782 & \pm 7 & $\begin{array}{ll}\text { (s.d.) } & (\mathrm{N}=9)^{* *}\end{array}$ & $J G 2 \cdot 1$ & $0.512218 \pm 13$ & & \\
\hline & & \pm 6 & $(2 \sigma \mathrm{m}) \quad(\mathrm{N}=9)^{*}$ & $\mathrm{JG} 2 \cdot 2$ & $0.512210 \pm 14$ & & \\
\hline & & & & $J G 2 \cdot 3$ & $0.512193 \pm 13$ & & \\
\hline & & & & $J G 2 \cdot 4$ & $0.512212 \pm 10$ & & \\
\hline JB2-1 & 0.513081 & \pm 15 & & $\mathrm{JG} 2.5$ & $0.512227 \pm 14$ & & \\
\hline $\mathrm{JB} 2 \cdot 2$ & 0.513087 & \pm 19 & & & $0.512212 \pm 12$ & (s.d.) & $(\mathrm{N}=5)^{* *}$ \\
\hline $\mathrm{JB} 2 \cdot 3$ & 0.513095 & \pm 14 & & & \pm 15 & $(2 \sigma \mathrm{m})$ & $(N=5)^{* *}$ \\
\hline JB2.4 & 0.513097 & \pm 14 & & & & & \\
\hline \multirow[t]{5}{*}{$\mathrm{JB} 2.5$} & 0.513089 & \pm 10 & & & & & \\
\hline & 0.513090 & \pm 6 & $\begin{array}{ll}\text { (s.d.) } & (\mathrm{N}=5)^{* *}\end{array}$ & JG3.1 & $0.512611 \pm 15$ & & \\
\hline & & \pm 8 & $(2 \sigma \mathrm{m}) \quad(\mathrm{N}=5)^{* *}$ & $\mathrm{JG} 3 \cdot 2$ & $0.512611 \pm 18$ & & \\
\hline & & & & JG3-3 & $0.512630 \pm 14$ & & \\
\hline & & & & $J G 3.4$ & $0.512631 \pm 11$ & & \\
\hline JB3-1 & 0.513030 & \pm 7 & & JG3.5 & $0.512600 \pm 13$ & & \\
\hline $\mathrm{JB} 3 \cdot 2$ & 0.513043 & \pm 8 & & & $0.512617 \pm 13$ & (s.d.) & $(\mathrm{N}=5)^{* *}$ \\
\hline JB3-3 & 0.513038 & \pm 11 & & & \pm 17 & $(2 \sigma \mathrm{m})$ & $(\mathrm{N}=5)^{* *}$ \\
\hline JB3-4 & 0.513035 & \pm 10 & & & & & \\
\hline \multirow[t]{5}{*}{ JB3.5 } & 0.513040 & \pm 8 & & & & & \\
\hline & 0.513037 & \pm 5 & (s.d.) $\quad(\mathrm{N}=5)^{* *}$ & JGb1-1 & $0.512647 \pm 13$ & & \\
\hline & & \pm 6 & $(2 \sigma \mathrm{m}) \quad(\mathrm{N}=5)^{* *}$ & JGb1-2 & $0.512628 \pm 12$ & & \\
\hline & & & & $J G b 1 \cdot 3$ & $0.512635 \pm 13$ & & \\
\hline & & & & JGb1.4 & $0.512667 \pm 16$ & & \\
\hline $\mathrm{JA1} \cdot 1$ & 0.513101 & \pm 10 & & $\mathrm{JGb1} \cdot 5$ & $0.512661 \pm 10$ & & \\
\hline $\mathrm{JA1} \cdot 2$ & 0.513090 & \pm 9 & & & $0.512648 \pm 16$ & (s.d.) & $(N=5)^{* *}$ \\
\hline $\mathrm{JAl} \cdot 3$ & 0.513103 & \pm 14 & & & \pm 20 & $(2 \sigma \mathrm{m})$ & $(\mathrm{N}=5)^{*}$ \\
\hline $\mathrm{JA1} \cdot 4$ & 0.513084 & \pm 7 & & & & & \\
\hline \multirow[t]{5}{*}{$\mathrm{JA} 1.5$} & 0.513081 & \pm 10 & & & & & \\
\hline & 0.513092 & \pm 10 & (s.d.) $\quad(\mathrm{N}=5)^{* *}$ & $\mathrm{JR} 1 \cdot 1$ & $0.512911 \pm 14$ & & \\
\hline & & \pm 12 & $(2 \sigma \mathrm{m}) \quad(\mathrm{N}=5)^{*}$ & $\mathrm{JR} 1 \cdot 2$ & $0.512912 \pm 14$ & & \\
\hline & & & & $\mathrm{JR} 1 \cdot 3$ & $0.512912 \pm 14$ & & \\
\hline & & & & $J R 1 \cdot 4$ & $0.512899 \pm 13$ & & \\
\hline $\mathrm{JA} 2 \cdot 1$ & 0.512518 & \pm 15 & & $\mathrm{JR} 1 \cdot 5$ & $0.512906 \pm 12$ & & \\
\hline $\mathrm{JA} 2 \cdot 2$ & 0.512528 & \pm 9 & & & $0.512908 \pm 6$ & (s.d.) & $(\mathrm{N}=5)^{* *}$ \\
\hline JA2.3 & 0.512538 & \pm 17 & & & \pm 7 & $(2 \sigma \mathrm{m})$ & $(\mathrm{N}=5)^{*}$ \\
\hline JA2.4 & 0.512540 & \pm 11 & & & & & \\
\hline \multirow[t]{5}{*}{$\mathrm{JA2} \cdot 5$} & 0.512525 & \pm 13 & & & & & \\
\hline & 0.512530 & \pm 9 & $\begin{array}{ll}\text { (s.d.) } & (\mathrm{N}=5)^{*}\end{array}$ & $J R 2 \cdot 1$ & $0.512909 \pm 11$ & & \\
\hline & & \pm 11 & $(2 \sigma \mathrm{m}) \quad(\mathrm{N}=5)^{*}$ & $\mathrm{JR} 2 \cdot 2$ & $0.512917 \pm 10$ & & \\
\hline & & & & $\mathrm{JR} 2 \cdot 3$ & $0.512903 \pm 13$ & & \\
\hline & & & & $J R 2 \cdot 4$ & $0.512927 \pm 12$ & & \\
\hline JA3.1 & 0.512855 & \pm 17 & & $\mathrm{JR} 2 \cdot 5$ & $0.512911 \pm 13$ & & \\
\hline JA3.2 & 0.512863 & \pm 14 & & & $0.512913 \pm 9$ & (s.d.) & $(\mathrm{N}=5)^{* *}$ \\
\hline JA3.3 & 0.512858 & \pm 11 & & & \pm 11 & $(2 \sigma \mathrm{m})$ & $(\mathrm{N}=5)^{*}$ \\
\hline JA3-4 & 0.512867 & \pm 11 & & & & & \\
\hline \multirow[t]{3}{*}{ JA3.5 } & 0.512851 & \pm 16 & & LaJolla & $0.511851 \pm 6$ & $(n=17)^{\circ}$ & \\
\hline & 0.512859 & \pm 6 & (s.d.) $\quad(\mathrm{N}=5)^{* *}$ & & & & \\
\hline & & \pm 8 & $(2 \sigma \mathrm{m}) \quad(\mathrm{N}=5)^{* *}$ & JNdi-1 & $0.512106 \pm 3$ & $(n=44)^{\circ}$ & \\
\hline
\end{tabular}

*Number of measurements; **Number of samples which are separately decomposed and extracted. s.d.: standard deviation. 
(1992), Iizumi et al. (1994) and Yamamoto and Maruyama (1996) reported Nd isotope values of GSJ-RRS with the value of La Jolla. The La Jolla values in their reports are $0.511864 \pm 0.000006$ $\left(2 \sigma_{\mathrm{m}}\right), 0.511848 \pm 0.000003\left(2 \sigma_{\mathrm{m}}\right)$ and 0.511829 $\pm 0.000024\left(2 \sigma_{\mathrm{m}}\right)$ respectively. Like ${ }^{87} \mathrm{Sr} /{ }^{86} \mathrm{Sr}$ ratios, our data were compared with the previously reported data normalized using La Jolla value of 0.511851 .

Previously reported Nd isotopic ratios for JB1a sample range from 0.512758 to 0.512807 (Arakawa, 1992; Iizumi et al., 1995; Yamamoto and Maruyama, 1996). Our average ${ }^{143} \mathrm{Nd} /{ }^{144} \mathrm{Nd}$ ratio of $0.512782 \pm 0.000006\left(2 \sigma_{\mathrm{m}}\right)$ lies within this range. Mean ${ }^{143} \mathrm{Nd} /{ }^{144} \mathrm{Nd}$ ratio of JB-3 is also within the range of previously reported data (0.513033 0.513038; Arakawa, 1992; lizumi et al., 1995). Our average ${ }^{143} \mathrm{Nd} /{ }^{144} \mathrm{Nd}$ ratio of JG-1a is within the deviation of the highest values previously reported $\left(0.512365 \pm 0.000018\left(2 \sigma_{\mathrm{m}}\right)\right.$; Arakawa, 1992).

Mean ${ }^{143} \mathrm{Nd} /{ }^{144} \mathrm{Nd}$ ratios of JA-2, JG-2 and JG3 obtained by us are close to the values reported by Arakawa (1992) (JA-2; $0.512537 \pm 0.000012$ $\left(2 \sigma_{\mathrm{m}}\right)$, JG-2; $0.512219 \pm 0.000007\left(2 \sigma_{\mathrm{m}}\right)$, JG-3; $\left.0.512606 \pm 0.000007\left(2 \sigma_{\mathrm{m}}\right)\right)$. But our mean Nd isotopic data for JB-2, JA-1, JGb-1 and JR-1 are higher than value reported by Arakawa (1992) (JB$2 ; 0.513042 \pm 0.000008\left(2 \sigma_{\mathrm{m}}\right), \mathrm{JA}-1 ; 0.513073 \pm$ $0.000005\left(2 \sigma_{\mathrm{m}}\right)$, JGb- $1 ; 0.512611 \pm 0.000008$ $\left.\left(2 \sigma_{\mathrm{m}}\right), \mathrm{JR}-1 ; 0.512893 \pm 0.000006\left(2 \sigma_{\mathrm{m}}\right)\right)$.

Neodymium isotopic ratios for JA-3 and JR-2 have not been previously reported, and the mean ${ }^{143} \mathrm{Nd} /{ }^{144} \mathrm{Nd}$ ratios of our measurements are $0.512859 \pm 0.000008\left(2 \sigma_{\mathrm{m}}\right)$ for JA-3 and 0.512913 $\pm 0.000011\left(2 \sigma_{\mathrm{m}}\right)$ for JR-2.

In summary, five to nine aliquots for each GSJRRS measurements reported here gave small standard deviations of both $\mathrm{Sr}$ and $\mathrm{Nd}$ isotopic ratios. Most $\mathrm{Sr}$ and $\mathrm{Nd}$ isotopic ratios reported here are within previously reported data range, but some present $\mathrm{Nd}$ isotopic ratios are higher than previously reported data.

Acknowledgments-We thank Prof. H. Kagami of Niigata University for his helpful suggestions during this study and reading of the manuscript. We are indebted to Dr. Paul A. Morris of the Geological Survey of Western Australia for reading and improving the manuscript. Thanks are also due to N. Kanazawa (Thermoquest Instruments Inc.) for his assistance in establishing mass spectrometric procedures in our laboratory. We especially acknowledge T. Takimoto (Niigata Univ.) who kindly carried out improvements in our chemical laboratory in which decomposition of samples and chemical separation were made.

\section{REFERENCES}

Ando, A. and Shibata, K. (1988) Isotopic data and rare gas compositions of GSJ rock reference samples, "Igneous rock series", 1988. Geochem. J. 22, 149156.

Arakawa, Y. (1992) ${ }^{143} \mathrm{Nd} /{ }^{144} \mathrm{Nd}$ ratios of twelve GSJ rock reference samples and reproducibility of the data. Geochem. J. 26, 105-109.

Iizumi, S., Maehara, K., Morris, P. A. and Sawada, Y. (1994) $\mathrm{Sr}$ isotope data of some GSJ rock reference samples. Mem. Fac. Sci. Shimane Univ. 28, 83-86.

Iizumi, S., Morris, P. A. and Sawada, Y. (1995) Nd isotope data for GSJ reference samples JB-1a, JB-3 and JG-1a and the La Jolla standard. Mem. Fac. Sci. Shimane Univ. 29, 73-76.

Kagami, H., Okano, O., Sudo, H. and Honma, H. (1982) Isotopic analysis of $\mathrm{Rb}$ and $\mathrm{Sr}$ using a full automatic thermal ionization mass spectrometer. Paper Inst. Therm. Spring Ress., Okayama Univ. No. 52, 51-70.

Kagami, H., Iwata, M., Sano, S. and Honma, H. (1987) $\mathrm{Sr}$ and $\mathrm{Nd}$ isotopic compositions and $\mathrm{Rb}, \mathrm{Sr}, \mathrm{Sm}$ and Nd concentrations of standard samples. Technical Rep. ISEI Okayama Univ. Ser. B, No. 4, 1-16.

Kagami, H. Yokose, H. and Honma, H. (1989) ${ }^{87} \mathrm{Sr} /{ }^{86} \mathrm{Sr}$ and ${ }^{143} \mathrm{Nd} /{ }^{144} \mathrm{Nd}$ ratios of GSJ rock reference samples; JB-1a, JA-1 and JG-1a. Geochem. J. 23, 209-214.

Kurasawa, H. (1984) Strontium isotopic consequence of the volcanic rocks from Fuji, Hakone and Izu area. Rept. Geol. Surv. Japan 35, 637-659 (in Japanese).

Lugmair, G. W. and Carlson, R. W. (1978) The SmNd history of KREEP. Proc. 9th Lunar Planet Sci. Conf. 689-704.

Nohda, S. and Wasserburg, G. J. (1981) Nd and Sr isotopic study of volcanic rocks from Japan. Earth Planet. Sci. Lett. 52, 264-276.

Okano, O., Kanazawa, R., Tosa, H. and Matsumoto, H. (1989) Sr, Nd and Ce isotopic measurements of GSJ standard rocks using MAT262 equipped with variable multi-collector system. Abstract 1989 Nihon Chikyu-Kagakukai Nenkai, 268 (in Japanese).

Shibata, K. and Adachi, M. (1972) Rb-Sr and K-Ar 
geochronology of metamorphic rocks in the Kamiaso conglomerate, Central Japan. J. Geol. Soc. Japan 78, 265-271.

Tanaka, T., Togashi, S., Kamioka, H., Amakawa, H., Kagami, H., Hamamoto, T., Yuhara, M., Orihashi, Y., Yoneda, S., Asahara, Y., Tanimizu, M., Dragusanu, C., Shimizu, H., Kunimaru, T., Takahashi, K. and Yanagi, T. (1997) JNdi-1: A new neodymium isotopic reference. Abstract 1997 Japan Earth and Planet. Sci. Joint. Meeting, 455 (in Japa- nese).

Yamamoto, M. and Maruyama, T. (1996) The Sr and $\mathrm{Nd}$ isotopic analysis and the quantitative analysis of $\mathrm{Rb}$ and $\mathrm{Sr}$ by using MAT261. Rep. of the Research Institute of Natural Resources, Mining College, Akita Univ. 61, 17-30 (in Japanese).

Zhang Zichao (1987) in Ando, A. and Shibata, K. (1988) Isotopic data and rare gas compositions of GSJ rock reference samples, "Igneous rock series". Geochem. J. 22, 149-156. 